\title{
La « nouvelle géographie " de Joel Kotkin : un modèle pour comprendre la morphologie urbaine des villes du savoir ${ }^{1}$
}

\author{
Rémy Tremblay ${ }^{2}$ \\ INRS-Urbanisation, Culture et Société
}

\section{Qui est Joel Kotkin ?}

$\mathcal{J}$ oel Kotkin n'est ni géographe, ni professeur d'université. Il est historien de formation, journaliste, consultant (www.joelkotkin.com) et auteur d'un ouvrage sur les villes américaines de la nouvelle économie ${ }^{3}$ dans lequel il suggère un modèle unique d'organisation spatiale pour ce type de ville. Kotkin est aussi de ces penseurs, futuristes et autres qui ont cette capacité hors du commun de «lire » les espaces urbains au point de remettre en question les théories et concepts des plus grands universitaires et ce, tout en faisant avancer

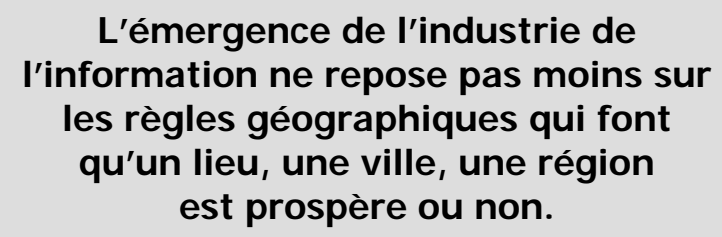

graphie économique et sociale des villes américaines à la suite de l'émergence de l'économie digitale (économie reliée à la haute technologie). Là où certains ont cru déceler l'avènement d'une société où la notion d'espace aurait disparu, Kotkin suppose que l'espace a plus d'importance que jamais. S'il est vrai qu'aujourd'hui les gens, les entreprises et les industries sont moins contraints de s'installer près des matières premières et des voies de transport, l'auteur nous rappelle que l'émergence de l'industrie de l'information ne repose pas moins sur les règles géographiques qui font qu'un lieu, une ville, une région est prospère ou non. Cette transcertaines disciplines. Par exemple, les géographes et sociologues « urbains » se rappelleront de l'impact majeur qu'a eu Joel Garreau, un journaliste du Washington Post, lorsqu'il a proposé le paradigme des « edge cities", ces villes périphériques qui ont poussées dans certaines régions métropolitaines américaines comme Washington, Houston et Los Angeles ${ }^{4}$. Dorénavant, tout manuel de géographie ou de sociologie urbaine se doit de citer Garreau.

Le modèle que propose Kotkin dans ses travaux apparaît, en théorie, fort original. D'autant qu'il répond à un besoin imminent de mieux comprendre l'organisation sociospatiale des villes du savoir, lesquelles villes répondent à des règles de localisation industrielle souvent très différentes de celles issues de l'économie «traditionnelle » ${ }^{5}$. En quoi consiste donc le modèle proposé par Joel Kotkin?

\section{Un modèle exclusif aux villes du savoir}

D'une part, Joel Kotkin analyse dans son ouvrage le nouveau visage, "The New Geography », de la géo- formation des notions de temps et d'espace rappelle l'apparition du chemin de fer et du télégraphe.

D'autre part, Kotkin nous suggère une modélisation de la morphologie urbaine des villes du savoir américaines. Selon lui, ces villes, somme toute nouvelles, comptent trois zones: 1) un «nerdistan», 2) une « midopolis » et 3) un centre.

Les " nerdistans », sont le résultat de la nouvelle économie, car c'est là que se trouvent les firmes de haute technologie (fabrication et R\&D) et le personnel qualifié, communément appelé talents. Les «nerdistans» sont généralement d'allure ultramoderne et de type campus, rappelant l'organisation spatiale des universités. Selon Joel Kotkin, les talents étant peu intéressés à la vie du centre-ville, ils souhaitent demeurer dans ces «nerdistans » dont l'urbanisme n'obéit qu'aux lois cartésiennes.

Bien que la nouvelle économie n'ait pas touché les au détriment de la classe moyenne, vu ces «midovieilles banlieues des villes du savoir, certaines ont, 
polis » passer par la gentrification. En effet, de nombreux talents ayant une famille, souvent d'origine non anglo-saxonne, ont opté pour la vie en condominium de luxe dans les «midopolis ». Cette zone a l'avantage d'être plus près de la ville et d'offrir un environnement socioculturel plus propice au style de vie familial. Il va sans dire qu'un tel chambardement n'est pas sans déplaire aux familles moyennes attachées depuis au moins une trentaine d'années à cette zone urbaine typiquement américaine et culturellement très homogène.

Quant aux centres de ces technopoles, ils accueillent, toujours d'après Kotkin, les «urbanites », lesquels représentent les nouveaux immigrés et les nouveaux urbains (couples sans enfants, célibataires, retraités, gais). Il arrive que des firmes de haute technologie choisissent même de s'y localiser. Les « urbanites » habitent des condos de luxe et ils préfèrent le centre pour le style de vie qu'il offre. Contrairement à ce que Richard Florida ${ }^{6}$ avance, ce ne sont pas toutes les technopoles qui jouissent d'un centre-ville animé et multiculturel. San Jose, au cœur de la Sillicon Valley, est tout simplement moribond. Raleigh, en Caroline du Nord, n'a rien d'une ville artistique et multiethnique. Austin au Texas, siège de la compagnie Dell, ne peut prétendre être plus animée et riche en histoire que sa consoeur texane San Antonio. Il n'en demeure pas moins que les technopoles américaines sont en grande majorité des villes dont le centre est propre et moderne, mais pas nécessairement sécuritaire ${ }^{7}$.

Les États-Unis ont vu des régions rurales tirer également profit de l'économie du savoir. Certaines, dont Park City dans l'Utah, se sont développées à tel point qu'elles sont devenues prospères et ont acquis une réputation enviable dans le domaine des nouvelles technologies. Joel Kotkin appelle ces régions des «Valhallas » ${ }^{8}$.

\section{Un modèle utile ?}

Le modèle de Kotkin nous apparaît théoriquement valable et attrayant. L'auteur suppose qu'il répond aux forces sociospatiales et économiques des régions métropolitaines de Raleigh-Durham, en Caroline du Nord, et de Los Angeles. Cependant, des études empiriques doivent être menées afin de vérifier la validité de ce modèle intriguant et prometteur.
Nos propres travaux sur Ottawa, une des principales technopoles nord-américaines, n'ont pas mis à épreuve le modèle de Kotkin ${ }^{9}$. Toutefois, il est permis de croire qu'il correspondrait fort bien à la morphologie urbaine de la Capitale canadienne. En effet, depuis une dizaine d'années, Ottawa s'est grandement transformée à la suite de la croissance fulgurante de la R\&D qui s'y déroule. Le centre-ville a vu naître nombre de services de même que plusieurs lofts et condos de luxe destinés aux talents. La «midopolis » d'Ottawa s'est gentrifiée et le « nerdistan », car il y en a un, est le lieu de résidence d'une grande proportion des quelque 50000 talents et des 1500 firmes de haute technologie, incluant Corel, Nortel et Alcatel. Mais, encore une fois, une étude empirique et une application cartographique ou schématique reste à faire et ce, même si tout semble être conforme au modèle de Kotkin.

\section{Des études empiriques doivent être menées afin de vérifier la validité de ce modèle intriguant et prometteur.}

Bref, Joel Kotkin nous offre un outil on ne peut plus géographique qui pourrait s'avérer très utile à ceux qui s'intéresse à l'organisation spatiale des villes du savoir.

\section{Notes et références}

1 Cette étude a été réalisée dans le cadre des activités de la Chaire de recherche du Canada en études urbaines et régionales, dont Mario Polèse, professeur à l'INRS, en est le titulaire.

2 Rémy Tremblay détient un doctorat en géographie de l'Université d'Ottawa. Il est chercheur postdoctoral à l'INRS-UCS et chargé de cours à l'UQO. Ses travaux portent sur les dimensions sociospatiales des villes nordaméricaines de l'économie du savoir.

3 Kotkin, J. (2000). The New Geography: How the Digital Revolution is Reshaping the American Landscape, New York, Random House.

4 Garreau, J. (1992). Edge City : Life on the New Frontier, New York, Anchor Books.

5 Porter, M.E. (1985). Competitive Advantage: Creating and Sustaining Superior Performance, New York, Free Press. 
6 Florida, R. (2001). The Rise of the Creative Class, New York, Basic Books.

7 Tremblay, R. (2004). « La place d'Ottawa-Gatineau parmi les villes du savoir nord-américaines », Organisations et territoires, vol. $13, \mathrm{n}^{\circ} 1$, p. 14-22.

8 Nous passerons outre les Valhallas puisque ce texte porte sur le modèle urbain de Kotkin.
9 Tremblay, R. (2003a). «Ottawa parmi les technopoles nord-américaines : perception des entrepreneurs locaux », Estudios Económico Regionales and Sectoriales/Regional and Sectoral Economic Studies, vol. 3, n ${ }^{\circ}$ 2, p. 29-40; Tremblay, R. (2003b). « Le tandem Gatineau-Ottawa ou la frontière fantôme », dans R. Côté et M. Venne (dir.), Québec 2003, Montréal, Fides, p. 322-327; Tremblay, R. (2004), op. cit. 


\section{Publicité}

« 4 carrés » de l’UQAC 